\title{
DEVELOPMENT OF A GREEN STABILITY-INDICATING HPLC-DAD METHOD FOR THE ANALYSIS OF TILDIPIROSIN IN PHARMACEUTICAL FORMULATION
}

\author{
MOHAMMED GAMAL ${ }^{1,2}$ \\ 'Department of Pharmaceutical Chemistry, Pharmacy College, Jouf University, \\ P.O. Box 2014, Sakaka, Aljouf, Kingdom of Saudi Arabia \\ ${ }^{2}$ Department of Pharmaceutical Analytical Chemistry, Faculty of Pharmacy, \\ Beni-Suef University, Alshaheed Shehata Ahmed Hegazy St., 62574 Beni-Suef, Egypt

\begin{abstract}
This study aimed to develop a simple stability-indicating HPLC-DAD method for the assay of Tildipirosin (TPS), which is a 16-member ring semi-synthetic macrolide, in an injectable solution. TPS is commonly used in the USA for the treatment of animal's respiratory infection. The isocratic elution was set up using the Inertsil-ODS column with the flow rate set at $0.9 \mathrm{~mL} / \mathrm{min}$ and the detection wavelength of $285 \mathrm{~nm}$. The column temperature was ambient and the overall runtime was $5 \mathrm{~min}$. Additionally, the mobile phase was water: acetonitrile: trifluoroacetic acid (53.45: $46.45: 0.10$, by volume). The HPLC-forced degradation stability heat, acid, base, and oxidation. The results showed the linearity range was 36.00 to $108.00 \mu \mathrm{g} / \mathrm{mL}$ for TPS. The correlation coefficient was higher than 0.999 and the accuracy was $98.78 \%$. The degradation percentages were presented ranging from $31.54 \%$ in the photodegradation condition to $50.10 \%$ in the base degradation. The Ecoscale score was about 82 points indicating an excellent green chromatographic technique. Therefore, the novel method was the first, green, stability-indicating chromatographic method for a TPS analysis with its degradation products. However, the major drawbacks of the new chromatographic method are the incomplete identification of degradation products and the inability to propose an appropriate mechanism for drug degradation.
\end{abstract} \\ method for TPS in Egydipiro Injectable Solution was performed in different stress conditions, i.e. light/UV, \\ stability method was unique in terms of simplicity and environment-friendly aspects. The new HPLC-DAD \\ Overall, the method can be employed to evaluate TPS stability under various storage conditions.
}

Keywords: HPLC, stability-indicating method, forced degradation, Tildipirosin, injectable solution

Tildipirosin (TPS) is a recently available macrolide antibiotic used for the treatment of respiratory tract infection of cattle and pigs and also possesses an antimalarial activity (1). TPS has the tradename of Zuprevo ${ }^{\mathrm{TM}}$ in the Netherlands and Egydipiro Injectable Solution ${ }^{\mathrm{TM}}$ in Egypt. Egydipiro has not yet been available in Egyptian veterinary pharmacies, as it is still in the process of seeking official approval in Egypt. The drug may be administered intramuscularly (IM) or subcutaneously (SC) but never via IV. TPS is used in the United States for the treatment of respiratory disease because it shows antimicrobial activity against Mannheimia haemolytica, Pasteurella multocida, and Histophilus somni (1) and it is restricted for animal use only and not for humans. Moreover, cattle intended for human consumption should not take the drug for at least 21 days before being butchered (2).
TPS is one of the unique semi-synthetic 16members macrolides with a tribasic $\mathrm{N}$-amino group that linked to the lactone ring; all three nitrogen atoms can acquire positive charges depending on the $\mathrm{pH}$ values of the solution and the interaction with proteins and biomolecules (2). TPS is characterized by amphoteric characters that enhance its activity. Therefore, it can dissolve easily in the aqueous plasma fluid and oily cell membranes. TPS is characterized by rapid onset of absorption and a high rate of tissue accumulation at the same time. TPS can easily penetrate the bacterial cell wall until combined to its target ribosome in cytoplasm. The drug is more active than other macrolides because the drug remains inside the bacterial cell as no efflux mechanism is happened owing to its above-mentioned chemical properties (2). All pharmacokinetics parameters in plasma, bronchial fluids and lung tis-

* Corresponding author: e-mail: mgamalm3000@yahoo.com 
sues for TPS were illustrated by Meng et al. in 2012 whereas TPS was quickly reached to the respiratory tract, while its elimination was relatively slow, up to 21 days. Therefore, the embargo period for the injected animal is 21 days before slaughter (3). The antimicrobial activity of TPS and the minimum inhibitory concentrations (MIC) were reported by Rose et al. in 2016 against different bacterial strains, i.e. Salmonella enterica in different $\mathrm{pH}$ conditions (4). The activity of TPS increased with decreasing $\mathrm{pH}$ from 7.3 to 6.7 .

The pharmaceutical formulation for TPS is Zuprevo $^{\mathrm{TM}}$ injectable solution in the USA; each $\mathrm{mL}$ of Zuprevo $^{\mathrm{TM}} 18 \%$ is claimed to contain $180 \mathrm{mg}$ of TPS free base in addition to many pharmaceutical ingredients (2). The empirical formula for TPS is $\mathrm{C}_{41} \mathrm{H}_{71} \mathrm{~N}_{3} \mathrm{O}_{8}$ and the molecular weight is $734 \mathrm{~g} / \mathrm{mol}$. The chemical structure of TPS is shown in Figure 1. Many analytical techniques were described for TPS analysis in biological fluids. Because of the high polarity of TPS like many aminoglycoside antibiotics, it cannot be analyzed directly by ultra-highperformance liquid chromatography-mass spectrometry (UHPLC-MS/MS). Therefore, the pretreatment step was used to decrease the peak splitting of TPS by the addition of an ion-pairing (sodium 1-heptanesulfonate) to the extract and not to the mobile phase. The ion-pairing process improved the peak shape and integration by keeping the drug in unionized form only (5). The method was very sensitive whereas it measures by nanoscale. However, it had the cons of complicated pretreatment step and the unavailability of such an expensive instrument for regular quality control analysis in many laboratories (5).

Many pharmacokinetics studies for TPS have been reported for the analysis of biological fluids and animal tissues (3, 6-8). All these methods were depending on TPS estimation by LC-MS/MS instrument, which is not commonly used, in the daily routine analysis. However, a recently published HPLC pharmacokinetic study (9) for TPS in goat's plasma was done successfully. The method was very sensitive as it can detect as low as $1 \mathrm{ng} / \mathrm{mL}$. The mobile phase was composed of acetonitrile and formic acid. C18 was the stationary phase and the detection wavelength was $289 \mathrm{~nm}$. The chromatographic conditions were repeated with a few modifications as in the reported HPLC method (10) by Lie et al. in 2018. The recorded linearity range was from 0.05 to $10 \mu \mathrm{g} / \mathrm{mL}$. Although the higher sensitivity of these two HPLC methods, none of them is a stability-indication method. For qualitative and quantitative analysis of TPS, a recently Ultra-performance Liquid chromatography-tandem High-Resolution Quadrupole Time-of-flight Mass Spectrometry (UPLC-Q-TOF-MS) method (11) was successfully used. The method is highly sensitive and the first one that measured the major five fragments for TPS to confirm its chemical structure. The method has the cons of a lack of simplicity for regular TPS analysis.

The HPLC instrument is the first used chromatographic technique for drug analysis in pharmaceutical formulations (12-17). HPLC is a commonly used method for drug testing in stability-indicating methods because of its simplicity and availability (18). HPLC was used before for stability testing of many drugs, i.e. hyoscine N-butyl bromide (19), nicotine (20), dronedarone hydrochloride (21), idebenone which is a coenzyme Q10-similar compound (22) and many others. HPLC linked to an electrochemical detector (HPLC-ECD) used as a stability-indicating method for Clarithromycin

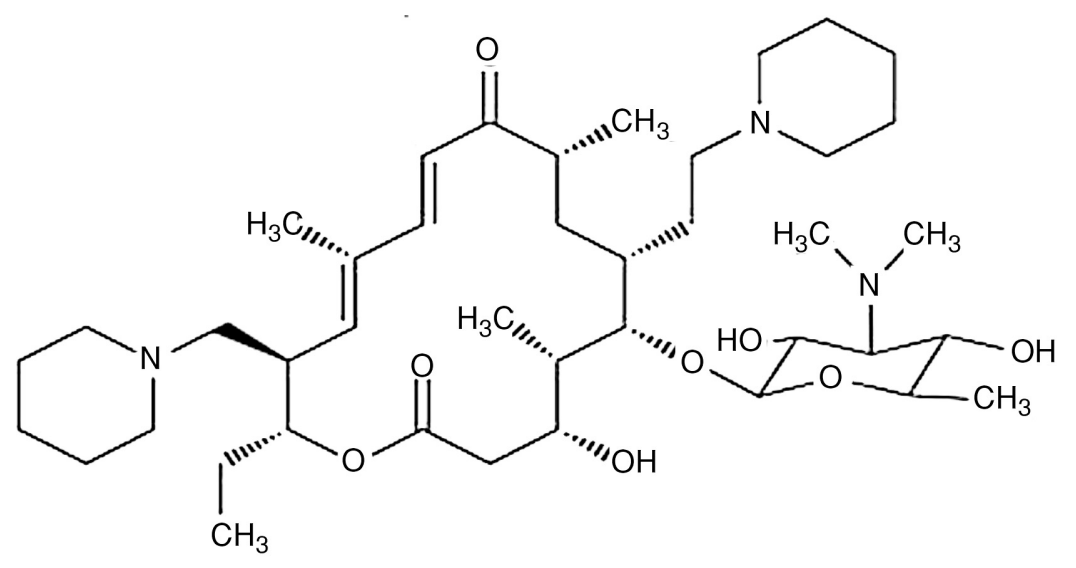

Figure 1. Chemical structures of Tildipirosin (TPS). 
analysis (23). This is one of the few reported stability HPLC methods for the estimation of a semi-synthetic macrolide. The method used an electrochemical detector because of the weak UV absorption spectrum for Clarithromycin. Moreover, Azithromycin in pharmaceuticals was estimated by HPLC with a UV detector at $215 \mathrm{~nm}(24), 210 \mathrm{~nm}(25)$ and $200 \mathrm{~nm}$ (26) in stability-indicating methods.

Up to now, no stability-indicating HPLC-DAD method has been reported for the routine analysis of TPS in pharmaceuticals. The main purpose of this study was thus to develop a simple, valid and accurate HPLC-DAD for the analysis of TPS, and to investigate TPS stability in different forced degradation conditions for selection of the proper storage conditions.

\section{EXPERIMENTAL}

\section{Chemicals and reagents}

Tildipirosin (TPS) pure powder, purity $=98 \%$, and Egydipiro Injectable Solution were kindly provided by the national organization for drug control and research (Cairo, Egypt) as a free sample. All used solvents (acetonitrile and trifluoracetic acid) were of gradient grade, $=99.9 \%$ for liquid chromatography and were purchased from Sigma Aldrich Chemie GmbH., (Steinheim, Germany). Deionized water was prepared via a water distillation system (Milli-Q) Merck (Darmstadt, Germany). The mobile phase was filtered and degassed before analysis via a $47 \mathrm{~mm}$ diameter Nylon filtration membrane $\left(\right.$ Allcrom $\left.{ }^{\circledR}\right)$.

\section{Chromatographic conditions}

The experiments were performed on Agilent 1260 Infinity II series, HPLC system, USA, supplied with a quaternary gradient pump model (G7111A) and autosampler model (G7129A). Chem station software UV detector model (G7115A) was used for the quantitative determination of TPS and its related degradation products. Many trails were evaluated to optimize the stability method. The best separation and acceptable chromatograms were accomplished by the application of the following chromatographic conditions that are summarized in Table 1.

\section{Preparation of standard solution}

- Stock solution (A): About $18 \mathrm{mg}$ of TPS was transferred to the 50-mL volumetric flask followed by $30 \mathrm{~mL}$ of mobile phase then the flask was sonicated for $10 \mathrm{~min}$. Finally, the flask was completed to $50 \mathrm{~mL}$ with the mobile solvent solution.

- Working solution (B): was prepared by dilution of stock solution A by the ratio of $10 \mathrm{~mL}$ : $50 \mathrm{~mL}$ solvent.

- Test solution (T1): was prepared by transferring $1 \mathrm{~mL}$ of solution for injection which is equivalent to $1.05 \mathrm{~g}$ of TPS and the same procedures in stock solution (A) were followed.

- Test solution (T2): was prepared by dilution of (T1) by the ratio $1 \mathrm{~mL}: 50 \mathrm{~mL}$ solvent.

\section{Procedures for forced degradation stability study for TPS}

The HPLC-forced degradation stability method $(18,27)$ for TPS in Egydipiro Injectable Solution was conducted in different stress conditions i.e. light/UV, heat, acid, base, and oxidation. Test solutions were prepared as follows:

A) Blank solution without any kind of treatment: The placebo was prepared by adding all inactive ingredients (citric acid monohydrate for adjusting of $\mathrm{pH}$, propylene glycol, and deionized water) as the ratio as in injectable solution without addition of TPS. Then follows as in test solutions

Table 1. The chromatographic conditions used in stability indicating HPLC method for TPS analysis.

\begin{tabular}{|c|c|}
\hline Column & Inertsil-ODS-3 C18, 250 X 4.6 mm, $5 \mu$ pore size equivalent \\
\hline Mobile phase & water : acetonitrile : trifluoroacetic acid $(53.45: 46.45: 0.1$, by Volume $)$ \\
\hline Detector & UV at $285 \mathrm{~nm}$ \\
\hline Pump mode & Isocratic \\
\hline Flow rate & $0.9 \mathrm{~mL} / \mathrm{min}$ \\
\hline Injection volume & $20 \mu \mathrm{L}$ \\
\hline Temperature & Ambient \\
\hline Run time & TPZ: 2.07 min; oxidative degradation products and base degradation products \\
\hline Retention times & $(\mathrm{A}=3.3, \mathrm{~B}=4.0$ min $) ;$ acid degradation products $(\mathrm{C}=3.0$ min $)$ \\
\hline
\end{tabular}


B) Light treatment: The working solution (B) was prepared as mentioned above and the flask was left under direct sunlight for $48 \mathrm{~h}$ and under UV light for $12 \mathrm{~h}$ then it was filtered through a syringe filter.

C) Heat treatment: The working solution (B) was prepared as mentioned above, and then, the flask was heated in a water bath at $80^{\circ} \mathrm{C}$ for $8 \mathrm{~h}$. After that, it was let to cool and filtered directly through a syringe filter.

D) Acid treatment: A volume of $5 \mathrm{~mL}$ of $1 \mathrm{~N} \mathrm{HCl}$ was added to $18 \mathrm{mg}$ TPS in $50 \mathrm{~mL}$-volumetric flask, and then the acidic flask was heated on a water bath at $80^{\circ} \mathrm{C}$ for one hour then the $\mathrm{pH}$ was adjusted to 7.0 with $1 \mathrm{~N} \mathrm{NaOH}$, followed by addition of $30 \mathrm{~mL}$ of the mobile phase and sonication for $10 \mathrm{~min}$ and the final volume was completed with the mobile phase. Then, proper dilution was done as in working solution (B).

E) Alkali treatment: A volume of $5 \mathrm{~mL}$ of $1 \mathrm{~N}$ $\mathrm{NaOH}$ was added to $18 \mathrm{mg}$ TPS in $50 \mathrm{~mL}$-volumetric flask, and then, the basic flask was heated on a water bath at $80^{\circ} \mathrm{C}$ for one hour then the $\mathrm{pH}$ was adjusted to 7.0 with $1 \mathrm{~N} \mathrm{HCl}$, followed by addition of $30 \mathrm{~mL}$ of the mobile phase and sonication for $10 \mathrm{~min}$. Finally, the volume was accomplished with the mobile phase. Then, proper dilution was done as in working solution (B).

F) $\mathrm{H}_{2} \mathrm{O}_{2}$ treatment: A volume of $5 \mathrm{~mL}$ of $0.5 \% \mathrm{H}_{2} \mathrm{O}_{2}$ was added to $18 \mathrm{mg}$ TPS in $50 \mathrm{~mL}$-volumetric flask, and then, the flask was heated on a water bath at $80^{\circ} \mathrm{C}$ for one hour then the $\mathrm{pH}$ was adjusted to 7.0 with $1 \mathrm{~N} \mathrm{NaOH}$, followed by addition of $30 \mathrm{~mL}$ of the mobile phase and sonication for $10 \mathrm{~min}$. finally, the volume was completed with the mobile phase. Then, proper dilution was done as in working solution (B).

\section{Estimation of validation parameters}

The validation parameters of the stability-indicating method i.e. accuracy, precision, linearity \& range, specificity, selectivity, ruggedness, detection and quantitation limits, and stability of solution were conducted according to the guidelines on analytical method validation ICH Q2(R1) (28).

\section{Evaluation of the method greenness value}

Analytical Eco-scale (29-32) for evaluation of the greenness of analytical technique was performed for calculation of the penalty points for evaluation of the whole system safety (including reagents, the type of chromatographic technique, occupational hazard and the presence of chemical wastes).

\section{RESULTS AND DISCUSSIONS}

The HPLC is generally preferred to the hyphenated LC-MS/MS technique in terms of instrument availability and simplicity. While the major advantages of the HPLC chromatographic methods are incomplete identification of degradation products and the inability to propose an appropriate mechanism for drug degradation. Moreover, the development of a green and simple chromatographic technique for the quality control of TPS is not a simple task because of the appearance of many degradates that interfere with TPS during the forced degradation study. The degradation of products in forced oxidation conditions was the same as in basic forced degradation conditions, while, the degradates in acidic forced degradation conditions were the same as in heat forced degradation conditions. Moreover, the forced light/UV degradation condition gave two characteristic peaks a major peak at $3 \mathrm{~min}$ and a minor peak at $4.7 \mathrm{~min}$. The new HPLC method was a simple, short run time (only $5 \mathrm{~min}$ ) and allows the separation, identification, and quantitative TPS determination as well as its impurities. In this research paper, a fast stability-indicating HPLC method for the quality control of TPS was developed and validated.

\section{Selection of optimum chromatographic condi- tions}

Column selection: Many analytical stationary phases were tested i.e. C18, C8 and cyano columns.

Table 2. The percentage of recoveries and degradations of TPS after forced degradation conditions.

\begin{tabular}{|c|c|c|c|c|}
\hline \multirow{2}{*}{$\begin{array}{c}\text { Mode of } \\
\text { degradation }\end{array}$} & Conditions & Area & Assay $\%$ & Degradation $\%$ \\
\cline { 3 - 5 } & Light $(48 \mathrm{~h}) / \mathrm{UV}(12 \mathrm{~h})$ & 2081.94 & $68.46 \%$ & $31.54 \%$ \\
\hline Light & $80^{\circ} \mathrm{C}(8 \mathrm{~h})$ & 1566.95 & $51.52 \%$ & $48.48 \%$ \\
\hline Heat & $1 \mathrm{~N} \mathrm{HCl} / 80^{\circ} \mathrm{C}(1 \mathrm{~h})$ & 2045.35 & $67.25 \%$ & $32.75 \%$ \\
\hline Acid & $1 \mathrm{~N} \mathrm{NaOH} / 80^{\circ} \mathrm{C}(1 \mathrm{~h})$ & 1517.74 & $49.90 \%$ & $50.10 \%$ \\
\hline Base & $0.5 \% \mathrm{H}_{2} \mathrm{O}_{2} / 80^{\circ} \mathrm{C}(1 \mathrm{~h})$ & 1786.65 & $58.75 \%$ & $41.25 \%$ \\
\hline $\mathrm{H}_{2} \mathrm{O}_{2}$ & & & & \multicolumn{3}{|c|}{. } \\
\hline
\end{tabular}


Table 3. The analytical validation parameters characteristics for TPS in stability indicating HPLC method with reference to ICH guidelines.

\begin{tabular}{|c|c|c|}
\hline Parameters & Values & Acceptance criteria \\
\hline Linearity \& range & 36.0 to $108.0 \mu \mathrm{g} / \mathrm{mL}$ & $\mathrm{rSD} \leq 2 \%$ \\
\hline Precision & 0.07 & $98-102 \%$ \\
\hline Accuracy & $98.78 \%$ & No interference \\
\hline Specificity / selectivity & $\begin{array}{c}\text { separated peak from degradation } \\
\text { products peaks }\end{array}$ & From the calibration curve: $3.3 \times \sigma / \mathrm{S}$ \\
\hline Limit of detection & 2.664 & From the calibration curve: $10 \times \sigma / \mathrm{S}$ \\
\hline Limit of quantitation & 8.073 & Pooled RSD $\leq 3 \%$ in every change item \\
\hline Ruggedness & $\begin{array}{c}0.39 \text { for different days } \\
0.32 \text { for different analysts }\end{array}$ & Pooled RSD $\leq 3 \%$ in every change item \\
\hline Robustness & 0.29 & \\
\hline
\end{tabular}

Table 4. The Linearity range for TPS in stability indicating HPLC method.

\begin{tabular}{|c|c|c|}
\hline \multicolumn{3}{|c|}{ Tildipirosin } \\
\hline Conc. $(\mu \mathrm{g} / \mathrm{mL})$ & Observed peak area & Average \\
\hline \multirow{3}{*}{36.00} & 1481.05 & \multirow{3}{*}{1481.93} \\
\hline & 1481.07 & \\
\hline & 1483.66 & \\
\hline \multirow{3}{*}{54.00} & 2299.67 & \multirow{3}{*}{2278.45} \\
\hline & 2268.79 & \\
\hline & 2266.89 & \\
\hline \multirow{3}{*}{72.00} & 3010.37 & \multirow{3}{*}{3015.87} \\
\hline & 3016.85 & \\
\hline & 3020.40 & \\
\hline \multirow{3}{*}{90.00} & 3706.34 & \multirow{3}{*}{3706.65} \\
\hline & 3710.71 & \\
\hline & 3702.89 & \\
\hline \multirow{3}{*}{108.00} & 4439.25 & \multirow{3}{*}{4440.02} \\
\hline & 4432.96 & \\
\hline & 4447.87 & \\
\hline \multicolumn{3}{|c|}{$\mathrm{R}=0.99970$} \\
\hline
\end{tabular}

Among these columns, Inertsil ODS-3 (C18, $250 \mathrm{x}$ $4.6 \mathrm{~mm}, 5 \mu \mathrm{m}$ pore size) gave the most acceptable chromatogram.

Mobile phase optimization: Water was chosen because of its greenness character. However, its safety, no separation was achieved without the addition of organic solvent and acid. The use of nonaqueous solvents with high eluotropic values greatly reduce the interactions of water with the column and improve the resolution values. Different organic solvents were tested i.e. methanol, ethanol, ethyl acetate, and acetonitrile. However, acetonitrile was relatively the less favored solvents in terms of greenness point of view $(14,31,32)$; it showed the best chromatogram in terms of peak symmetry and baseline stability. Therefore, acetonitrile was chosen as an organic solvent with water. Because of the alkalinity of tribasic TPS, it was necessary to add acid to the mobile phase. Moreover, different acids were also tested, i.e. formic acid (FA), acetic acid (AC) and trifluoroacetic acid (TFA). TFA (15) improved the peak symmetry and it was our choice. It seems that TFA made ion pairing with the tribasic 16-member ring of macrolide antibiotic. Finally, the optimum mobile phase was a mixture of water: acetonitrile: trifluoroacetic acid $(53.45: 46.45: 0.10$, by volume).

Wavelength selection: Different wavelengths were experienced, i.e. 210, 250, and $285 \mathrm{~nm}$. The highest sensitivity was observed at $285 \mathrm{~nm}$. Therefore, it was chosen for the development of the method.

Flow rate choice: Many trails were done to select the most suitable flow rate that achieves an adequate resolution in the least possible time. The best results were recorded when the flow rate was $0.9 \mathrm{~mL} / \mathrm{min}$. Therefore, it was chosen for the development of the method.

Effect of column temperature on the chromatogram: The temperature was kept at ambient temperature, which was around $28^{\circ} \mathrm{C}$. The conditions set for the method development were reported before in Tables 1 and 2 .

\section{Material and methods}

\section{Forced degradation conditions}

The peak of TPS is well resolved from other peaks for the degradation products which appeared after treatment with different forced degradation conditions, i.e. UV light, base, heat, acid hydrolysis, 
Table 5. The accuracy results for TPS in stability indicating HPLC method.

\begin{tabular}{|c|c|c|c|}
\hline Conc $(\%)$ & Observed peak area & Average & Recovery (\%) \\
\hline \multirow{3}{*}{$50 \%$} & 1512.29 & \multirow{3}{*}{1513.16} & \multirow{3}{*}{$99.51 \%$} \\
\hline & 1513.84 & & \\
\hline & 1513.36 & & \\
\hline \multirow{3}{*}{$100 \%$} & 2992.39 & \multirow{3}{*}{2996.65} & \multirow{3}{*}{$98.53 \%$} \\
\hline & 2999.57 & & \\
\hline & 2997.98 & & \\
\hline \multirow{3}{*}{$150 \%$} & 4485.17 & \multirow{3}{*}{4483.83} & \multirow{3}{*}{$98.29 \%$} \\
\hline & 4484.29 & & \\
\hline & 4482.02 & & \\
\hline \multicolumn{3}{|c|}{ Maximum recovery } & $99.51 \%$ \\
\hline \multicolumn{3}{|c|}{ Minimum recovery } & $98.29 \%$ \\
\hline \multicolumn{3}{|c|}{ Average } & $98.78 \%$ \\
\hline
\end{tabular}

Table 6. The precision results for TPS in stability indicating HPLC method.

\begin{tabular}{|c|c|c|}
\hline \multirow{2}{*}{ Determination No. } & Observed peak Area & \multirow{2}{*}{ Acceptance criteria } \\
\cline { 2 - 2 } & Tildipirosin & \multirow{2}{*}{} \\
\hline 1 & 3040.03 & \\
\hline 3 & 3044.24 & \\
\hline 4 & 3039.89 & \\
\hline 5 & 3038.24 \\
\hline 6 & 3042.73 \\
\hline Mean & 3042.61 & \\
\hline SD & 3041.29 & RSD $\leq 2 \%$ \\
\hline RSD & 2.25 & \\
\hline
\end{tabular}

Table 7. The specificity results for TPS in stability indicating HPLC method.

\begin{tabular}{|c|c|c|c|}
\hline Test name & Concentration $(\mu \mathrm{g} / \mathrm{mL})$ & Peak RT & Peak area \\
\hline Standard & 72.00 & 2.068 & 3029.04 \\
\hline Test & 72.00 & 2.067 & 2989.23 \\
\hline Placebo & 0 & No peak was observed & No peak was observed \\
\hline
\end{tabular}

Table 8 . The method's inter-day precision results (day to day) for TPS in stability indicating HPLC method.

\begin{tabular}{|c|c|c|c|}
\hline \multirow{2}{*}{ Replicate } & \multicolumn{2}{|c|}{ Tildipirosin } & \multirow{2}{*}{ Acceptance criteria } \\
\hline & Day 1 & Day 2 & \\
\hline 1 & 2976.45 & 2995.26 & \\
\hline 2 & 2985.60 & 3001.84 & \\
\hline 3 & 2977.87 & 3003.32 & \\
\hline Pooled mean & \multicolumn{2}{|c|}{2990.14} & \\
\hline Pooled SD & \multicolumn{2}{|c|}{11.82} & \\
\hline Pooled RSD & \multicolumn{2}{|c|}{0.39} & $\leq 3 \%$ \\
\hline
\end{tabular}


and hydrogen peroxide oxidation. The degradation products for the forced oxidation condition were the same as in the basic forced degradation condition whereas two similar peaks A and B were observed at retention times 3.3 and $4.0 \mathrm{~min}$, respectively. While the degradates in the acidic forced degradation condition were the same as in heat forced degradation condition whereas, a new peak $\mathrm{C}$ was recorded at $3.0 \mathrm{~min}$. Finally, the forced light/UV degradation condition gave two distinguished peaks; major peak $\mathrm{C}$ at a retention time $3.0 \mathrm{~min}$ and minor peak $\mathrm{D}$ with a retention time of $4.7 \mathrm{~min}$. The percentage of degradations are listed in Table 2. The maximum degradation was observed in the base degradation condition (with 50\%) while the minimum degradation was recorded in light/UV degradation conditions (with $31.54 \%$ ).

\section{Method validation}

The analytical procedure developed for the analysis of TPS was validated in accordance with ICH Q2(R1) in terms of linearity, limits of detection and quantification, precision, accuracy, specificity, and robustness (26). The numerical values of all validation parameters were in acceptable limits with referring to ICH guidelines, these results are discussed collectively in Table 3 . The stability of the solution was also evaluated. The specificity of the HPLC method was demonstrated by the single peak for TPS in the sample's degradation in acid, alkaline, thermic, oxidant and photodegradation conditions. This demonstrates the stability-indicating property of the novel HPLC-DAD method. Linearity was performed by preparing five different concentrations of TPS standards using the optimum chromatographic conditions as in Table 1. The linearity of TPS was in the range from 36.0 to $108.0 \mu \mathrm{g} / \mathrm{mL}$ as in Figure 2 . The correlation coefficient, which was $=0.99$, covered from $50 \%$ to $150 \%$ of the standard solution after proper dilution. Linearity data were shown in Table 4. The y-intercept was statistically equal to 46.827 .

For estimation of accuracy of the chromatographic method, the samples were spiked by the addition of well-known quantities of the standard to the placebo matrix containing all excipients as in the final pharmaceutical product. Accuracy is assessed using three determinations over certain concentrations which is from $50 \%$ to $150 \%$, including $100 \%$ of the target concentration $(72.00 \mu \mathrm{g} / \mathrm{mL})$. As illustrated in Table 5 , the percentages of recoveries were

Table 9. The method reproducibility results (analyst to analyst) for TPS in stability indicating HPLC method.

\begin{tabular}{|c|c|c|c|}
\hline \multirow{2}{*}{ Replicate\# } & \multicolumn{2}{|c|}{ Tildipirosin } & \multirow{2}{*}{ Acceptance criteria } \\
\cline { 2 - 3 } & Analyst 1 & Analyst 2 & \multirow{2}{*}{3048.58} \\
\hline 1 & 3071.74 & 3049.86 & \\
\hline 2 & 3068.01 & 3054.48 & \\
\hline 3 & 3063.25 & \\
\hline Pooled mean & \multicolumn{2}{|c|}{3059.32} & \\
\hline Pooled SD & \multicolumn{2}{|c}{0.32} & $\leq 3 \%$ \\
\hline Pooled RSD & \multicolumn{2}{|c|}{} \\
\hline
\end{tabular}

Table 10. The robustness results (slight changes in mobile phase composition) for TPS in stability indicating HPLC method.

\begin{tabular}{|c|c|c|c|}
\hline \multirow{2}{*}{ Replicate\# } & \multicolumn{2}{|c|}{ Tildipirosin } & \multirow{2}{*}{ Acceptance criteria } \\
\cline { 2 - 3 } & Condition 1 & Condition 2 & \multirow{2}{*}{3051.62} \\
\hline 1 & 3050.98 & 3034.83 & \\
\hline 2 & 3046.26 & 3035.48 & \\
\hline 3 & 3056.45 & \\
\hline Pooled mean & \multicolumn{2}{|c|}{3045.93} & \multirow{2}{|c}{$\leq 3 \%$} \\
\hline Pooled SD & \multicolumn{2}{|c|}{0.29} & \\
\hline Pooled RSD & & \\
\hline
\end{tabular}

Condition 1: water : acetonitrile : trifluoroacetic acid ( $53.45: 46.45: 0.1$, by Volume); Condition 2: water : acetonitrile : trifluoroacetic acid ( $50.45: 49.45: 0.1$, by Volume). 
between 98.00 and $102.00 \%$; therefore, the method is accurate. The precision is conducted using six replicates of the same standard solution (72.00 $\mu \mathrm{g} / \mathrm{mL}$ ). The precision of the analytical method was evaluated as repeatability. The RSD was below $2 \%$, and the system suitability parameters were achieved. The results were shown in Table 6. The method specificity was determined for TPS standard samples and placebo of Egydipiro Injectable Solution, the chromatograms showed no interference from the forced degradation products. The data for method specificity was collectively summarized in Table 7 .

The ruggedness of the stability-indicating method was conducted by the analysis of the samples under a variety of conditions, such as different ana-

Table 11. Calculation of penalty points for evaluation of the greenness of the proposed HPLC-DAD method for the PTS analysis.

\begin{tabular}{|l|c|}
\hline Method parameters & Points \\
\hline 1- water & 0 \\
\hline 2- acetonitrile & 8 \\
\hline 3- trifluoroacetic acid & 2 \\
\hline HPLC & 0 \\
\hline Waste & 8 \\
\hline Occupational hazard & 0 \\
\hline Total penalty points & 18 \\
\hline Analytical Eco- scale score* & 82 \\
\hline
\end{tabular}

*Analytical Eco-scale score, ideal method if $=100$, excellent $>75$, acceptable $>50$, inadequate $<50$ lysts and different days, etc. The method's inter-day precision was performed by analysis of three replicates of a single concentration $(72.00 \mu \mathrm{g} / \mathrm{mL})$ on the first day, and then on a second day. The replicates of the freshly prepared samples were analyzed and the equivalent concentrations were calculated. The results of day to day recoveries were illustrated in Table 8 . Moreover, the method reproducibility was performed by evaluating the results from two different analysts, as in Table 9, whereas, two identical samples were prepared by two analysts and analyzed using the same chromatographic conditions. Both Table 8 and Table 9 confirmed that the HPLC method is rugged as the pooled RSD is not more than $3 \%$. In addition, the robustness of the chromatographic method was conducted by the analysis of the samples under a variety of conditions, such as different in $\mathrm{pH}$ of mobile phase (condition 1) and a slight change in the mobile phase composition (condition 2). Three replicates of a single concentration were prepared and analyzed by the same analyst as the previously mentioned circumstances 1 and 2 . The results were recorded in Table 10. The HPLC method is robust as the pooled RSD is not more than $3 \%$.

The limits of detection and quantitation (LOD and LOQ) were assessed using the data of slope and $\mathrm{SD}$ in the calibration curve, Figure 2 and later confirmed by injecting the calculated concentrations at $2.664 \mu \mathrm{g} / \mathrm{mL}$ and $8.073 \mu \mathrm{g} / \mathrm{mL}$, respectively. Detailed descriptions of the equations used for the calculation of LOD and LOQ are given in Table 3. The stability of the PTS solution was monitored at room temperature for $48 \mathrm{~h}$, and the \% RSD values of

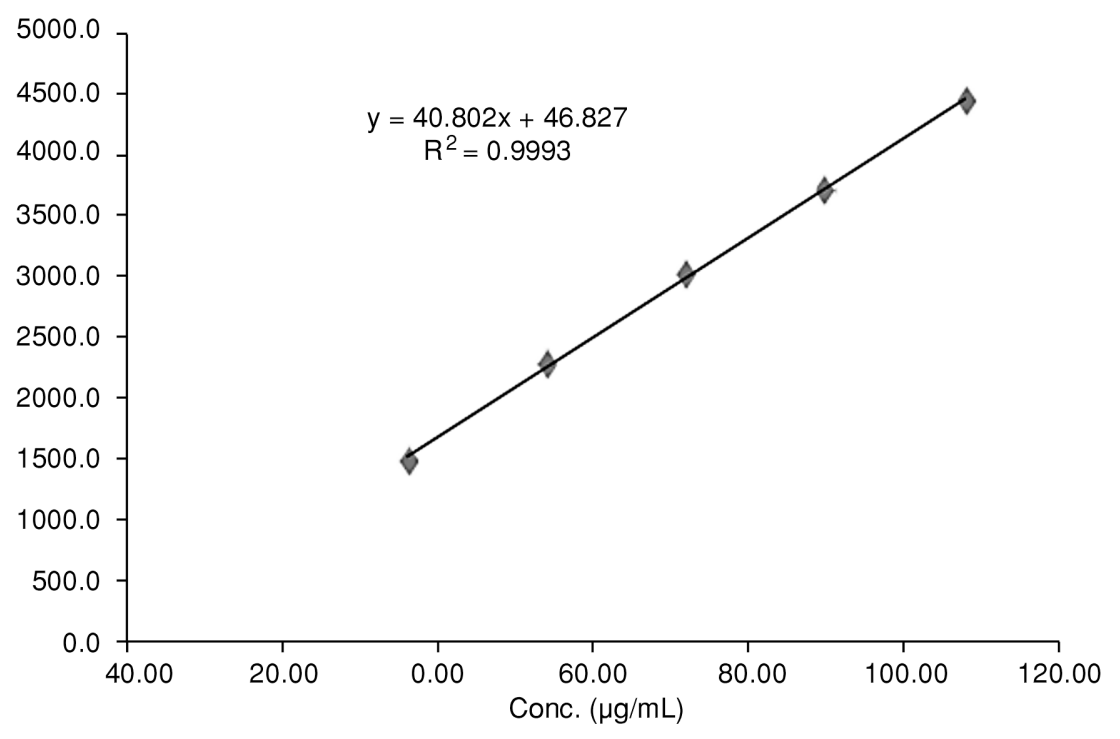

Figure 2. The calibration curve of Tildipirosin (TPS) with linearity equation. 
the peak areas were recorded as in Table 8. No significant changes were observed for the measured concentration $(72.00 \mu \mathrm{g} / \mathrm{mL})$ during the experimental period.

\section{Evaluation of method greenness}

The ideal solvent in the analytical Eco-scale point of view should have a value of 100 ; water is the ideal solvent in terms of safety without consideration of its concentration in the mobile phase. This means that the total penalty points that express the toxic effect of the stability method equal to 0 points, as shown in the equation below:

Analytical Eco-scale $=100-$ the sum of penalty points $(29,30)$

The average penalty points depend on the type of used solvents, their proportions, type of analytical method, presence of waste and occupational hazard. The method is evaluated as excellent $>75$, acceptable $>50$, inadequate $<50$. The Eco-scale score for the chromatographic methods was 82 PTS, as illustrated in Table 11, therefore, the newly HPLC-DAD technique is an excellent one in terms of the greenness approach.

\section{Limitations of the study and future research}

The method was not devised to analyze TPS in biological fluids because the main target for this research was to develop a simple and valid stabilityindicating HPLC chromatographic method for TPS analysis in systematic storage conditions. Owing to the shortage of active hyphenated techniques in Jouf University [LC-MS, TLC-MS], no mass spectrophotometric technique was done for the structure determination of degradation products. However, it is strongly recommended to reevaluate the forced degradation study for TPS by LC-MS to propose a degradation mechanism of TPS and complete the impurity profile of all degradation products. Furthermore, the refractive index and/or corona spray detectors should be tested to develop more sensitive HPLC methods. Last, TPS stability should be tested in diluted solutions $(0.1 \mathrm{~N})$ of acid and base. According to the overall findings, TPS should be protected from direct light, heat, oxidative, acid, and base conditions during its storage.

\section{CONCLUSION}

In this study, a straightforward, fast, and green HPLC-DAD method was optimized and validated for the quantification of TPS in both pure form and injection dosage form. The validation of stability indicating method was performed as guided by the ICH instructions. The proposed analytical method of TPS in Egydipiro Injectable Solution was precise, linear, accurate, rugged, selective and sensitive. Additionally, it was observed that TPS was liable to degradation under heat, light, oxidative, acidic, and alkaline conditions. No interference was found from variable degradation products in all forced degradation conditions on the main peak of TPS. Finally, the results confirmed that the new chromatographic method is appropriate for its intended purpose and could be used effectively in routine quality control analysis for TPS.

\section{Acknowledgment}

The author would like to acknowledge the technical support from Jouf University, (Aljouf, Saudi Arabia) and also the assistance of Dr. Win Watjana in editing the manuscript.

\section{Conflict of interests}

The author declares no conflict of interest.

\section{REFERENCES}

1. Arsic B., Barber J., Čikoš A., Mladenovic M., StankovicN., Novak P.: Int. J. Antimicrob. Agents 51, 283 (2018).

2. Overview - Zuprevo I Merck Animal Health USA, https://www.merck-animal-health-usa. com/zuprevo/, (accessed 18.02.2020).

3. Menge M., Rose M., Bohland C., Zschiesche E., Kilp S., et al.: J. Vet. Pharmacol. Ther. 35, 550 (2012).

4. Rose M., Pridmore A., Shaw A., Wilhelm C., Menge M., et al.: J. Vet. Pharmacol. Ther. 39, 277 (2016).

5. Lehotay S.J., Lightfield A.R.: Anal. Bioanal. Chem. 410, 1095 (2018).

6. Torres F., Santamaria R., Jimenez M., Menjón R., Ibanez A., et al.: J. Vet. Pharmacol. Ther. 39, 199 (2016).

7. Rose M., Menge M., Bohland C., Zschiesche E., Wilhelm C., et al.: J. Vet. Pharmacol. Ther. 36, 140 (2013).

8. Zeng D., Sun M., Lin Z., Li M., Gehring R., Zeng Z.: Front. Microbiol. 9, 1038 (2018).

9. Elazab S.T., Badawy M.E.: Jpn. J. Vet. Res. 68, 5 (2020).

10. Lei Z., Liu Q., Yang B., Ahmed S., Cao J., He Q.: Oncotarget 9, 1673 (2018). 
11. Wu J., Liu M., Shang F., Zhang C., Song M., et al.: Chiang Mai J. Sci. 44, 956 (2017).

12. Wageih Ali N., Gamal M., Abdelkawy M.: Pak. J. Pharm. Sci. 26, 865 (2013).

13. El-Saharty Y.S., Metwaly F.H., Refaat M., ElKhateeb S.Z.: Talanta 72, 675 (2007).

14. Gamal M., Ali H.M., Abdelfatah R.M., Magdy M.A.: Microchem. J. 151, 104258 (2019).

15. Ali N.W., Gamal M., Abdelkawy M.: Arab. J. Chem. 10, S1868 (2017).

16. Ali N.W., Gamal M., Abdelkawy M.: IOSR J. Pharm. Biol. Sci. 3, 29 (2012).

17. Gamal M., Ali H.M., Fraihat S.M., Seaf Elnasr T.A.: Luminescence 34, 644 (2019).

18. Gamal M.: Analyst 145, 2025 (2020).

19. Ali N.W., Gamal M.: Pharm. Anal. Acta 7, 2 (2013).

20. Bansal M., Sharma M., Bullen C., Svirskis D.: Int. J. Environ. Res. Public Health 15, 1737 (2018).

21. Dabhi B., Jadeja Y., Patel M., Jebaliya H., Karia D., Shah A.: Sci. Pharm. 81, 115 (2013).

22. Kombath S., Balde I.B., Carret S., Kabiche S., Cisternino S., et al.: J. Anal. Methods Chem. 2015, 5 pages (2015), doi:10.1155/2015/ 835986.
23. Makoni P.A., Chikukwa M.T.R., Khamanga S.M.M., Walker R.B.: Sci. Pharm. 87, 31 (2019).

24. Okaru A.O., Abuga K.O., Kamau F.N., Ndwigah S.N., Lachenmeier D.W., Pharmaceutics 9, 11 (2017).

25. Ghari T., Kobarfard F., Mortazavi S.A.: Iran. J. Pharm. Res. 12, 55 (2013).

26. Housheh S.: Asian J. Pharm. Res. 7, 55 (2017).

27. Bajaj S., Sakhuja N., Singla D.: J. Appl. Pharm. Sci. 2, 129 (2012).

28. Swartz M.E., Krull I.S.: Handbook of Analytical Validation, 2012.

29. Gałuszka A., Migaszewski Z.M., Konieczka P., Namieśnik J.: TrAC - Trends Anal. Chem. 37, 61 (2012).

30. Gamal M., Ali H.M., Abdelfatah R.M., Magdy M.A.: Microchem. J. 151, 104258 (2019).

31. Płotka-Wasylka J., Namieśnik J. (Eds.): Green Analytical Chemistry: Past, Present and Perspectives, Springer, 2019.ț

32. Płotka-Wasylka J., Fabjanowicz M., Kalinowska K., Namieśnik J.: History and milestones of green analytical chemistry. In: Green Analytical Chemistry, pp. 1-17, Springer, Singapore 2019.

(C) 2020 by Polish Pharmaceutical Society. This is an open-access article under the CC BY NC license (c) (1) (9) (http://creativecommons.org/licenses/by-nc/4.0/). 